\title{
The value of experimental models in the prophylaxis of bacterial endocarditis
}

\author{
R. Malinverni and M. P. Glauser* \\ Medizinische Universitätsklinik, Inselspital, CH-3010 Bern and *Division des Maladies Infectieuses, Centre \\ Hospitalier Universitaire Vaudois, CH-1011 Lausanne, Switzerland
}

KEY WORDS: Experimental endocarditis, natural history, clinical relevance for prophylaxis.

\section{Introduction}

Infective endocarditis (IE) is still a serious medical problem. It not only carries significant mortality during the actual period of endocardial infection, but causes morbidity that may persist beyond the duration of active infection. Complications such as heart failure, neurological deficits following central nervous system embolism, complications of heart surgery or anticoagulation following valve replacement are considerable. Thus it is clear that everything should be done to prevent IE. However, the development of prophylactic measures implies a precise knowledge of the pathogenesis of this disease.

Unfortunately, many questions regarding the pathogenesis and the prophylaxis of IE in humans remain unanswered; these include the precise sequence of events in the establishment of human endocarditis as well as the actual risk of a given invasive procedure to cause transient bacteraemia and endocarditis. Moreover, even the actual risk of development of endocarditis for a given underlying cardiac condition is uncertain and controversial. These and similar questions could only be answered by controlled clinical trials. However, it is unlikely that such clinical studies will be performed, mainly because the number of patients required would be too large ${ }^{11}$. One approach to partially solving these questions is a better understanding of the pathogenesis of IE as seen in experimental models; this may lead to a better rationale for prophylactic recommendations in man.

\section{Description of experimental models}

Freedman and coworkers originally developed a rabbit model of endocarditis whose rationale was derived from clinical observations ${ }^{|2|}$. They noted the ease with which intravenous polyethylene catheters became secondarily infected, resulting in bacteraemia in several patients. They also observed that their patients with catheter-induced sepsis had developed infective endocarditis at the point of contact of the indwelling central venous catheter in the right auricle. They therefore introduced a polyethylene catheter into the right and left heart of rabbits, filling the catheter with microorganisms. Durack et al ${ }^{[3]}$ changed the model somewhat in that they injected the bacteria intravenously, which resulted in a high incidence of tricuspid or aortic valve endocarditis ${ }^{(4)}$. Following a similar technique we have recently developed a model in rats, which will be briefly described ${ }^{|5|}$.

A polyethylene catheter is inserted across the aortic valve through the right carotid artery, resulting in the production of sterile valvular vegetations. These endocardial lesions resemble those found in humans after diseases such as rheumatic fever ${ }^{[3,6]}$. Twenty-four hours after catheterization rats are injected in the tail vein with a given bacterial inoculum of the test organism. The rats are sacrificed at varying time intervals after i.v. bacterial challenge; quantitative blood cultures are then drawn, the aortic vegetations are excised, weighed, homogenized, serially diluted and plated. Plates are counted after $48-72$ hours of incubation.

Antibiotic prophylaxis is performed by injecting one group of rats with a given dosage of the selected antibiotic at different time intervals before bacterial challenge. The dosage and time intervals are chosen so as to result in peak serum levels in rats similar to those in humans after a recommended dose at the time the organisms are injected.

The relevance of these experimental models to the human situation has been questioned mainly for two reasons $^{[3]}$. 


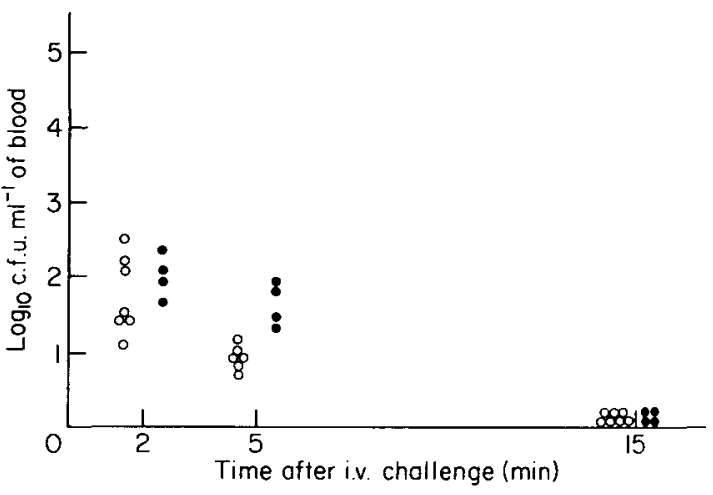

Figure 1 Quantitative determination of bacterial numbers circulating in the blood $\left(\log _{10}\right.$ c.f.u. $\left.\mathrm{ml}^{-1}\right)$ of rats at different times after i.v. challenge with $10^{4}$ c.f.u. of either $S$. intermedius (O), a viridans streptococcal strain, or S. faecalis 1209 (e). Each circle represents values from one rat.

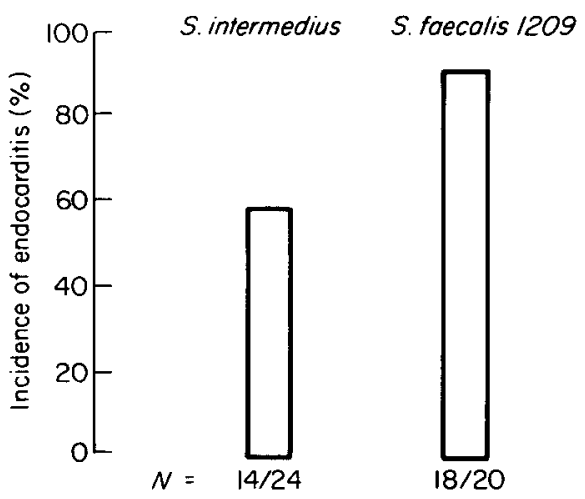

Figure 2 Incidence of endocarditis in rats 3 days after i.v. challenge with $10^{4}$ c.f.u. of either $S$. intermedius or $S$. faecalis 1209 . The number of rats with infected vegetations/total number of injected rats is indicated at the base of each column.

\section{THE RELEVANCE OF THE INTRACARDIAC CATHETER}

In most experimental studies the catheter has been left in place throughout the whole experiment, while most frequently there is no intravascular foreign body present in humans. There is experimental evidence, however, that the persistence of the catheter favours the development of $1 E^{[8.9]}$, and that antibiotic prophylaxis is facilitated when the catheter is removed $^{[5]}$. Thus, experimental models for the prophylaxis of endocarditis with a catheter in place provide a very stringent test of antibiotic prophylaxis. Furthermore, one may argue that it mimics clinical situations in humans with prosthetic heart valves or other intravascular foreign bodies.
THE MAGNITUDE OF THE BACTERAEMIA USED FOR BACTERIAL CHALLENGE

The magnitude of the bacterial inocula injected i.v. to the animals $\left(10^{4}-10^{8}\right.$ c.f.u.) has been considered irrelevant when compared to bacteraemias observed in humans. Indeed, the magnitude of bacteraemia observed in humans after certain procedures such as teeth extractions is generally of the order of $10^{1}-10^{2}$ c.f.u. $\mathrm{ml}^{-1}$ of blood for a given $\operatorname{strain}^{[10]}$. However, it must be kept in mind that i.v. bacterial challenge in rats results in circulating bacterial numbers that are far below the originally injected inoculum, due both to a passive haemodilution effect and to an active clearance mechanism by the reticulo-endothelial system ${ }^{[1]}$. Furthermore, it must be emphasized that the relationship between the magnitude of bacteraemia and the risk of subsequent development of IE in humans is unknown. Indeed, it is quite conceivable that those very patients who develop endocarditis after a given procedure are precisely those who presented the highest number of circulating bacteria.

\section{The properties of various bacterial strains to induce endocarditis}

The relationship between the magnitude of transient bacteraemia and the subsequent development of IE has been carefully investigated in the rat model of IE. It has been shown that for a given i.v. inoculum size of a test strain, the incidence of infection 3 days after challenge was fairly constant and reproducible. Wide variations have been observed between different bacterial strains in their ability to induce endocarditis $\left.{ }^{12} 14\right]$. Thus, the injection of similar numbers of various strains does not induce ipso facto the same incidence of endocarditis, although it is likely to induce similar magnitudes of bacteraemias. As an example, Fig. I shows the results of quantitative blood cultures performed in groups of rats injected with $10^{4}$ c.f.u. of either an $S$. faecalis strain or a viridans streptococcal strain ( $S$. intermedius). As can be seen, a similar number of $S$. faecalis and $S$. intermedius colonies were circulating at different times after i.v. challenge and all blood cultures were sterile $15 \mathrm{~min}$ after injection. Figure 2 shows the incidence of IE due to both strains 3 days after bacterial challenge. While the $S$. faecalis strain repeatedly infected $90 \%$ of the animals (ID 90), only about one-half $(58 \%)$ of the animals had $S$. intermedius endocarditis. Since the clearance of both organisms from the blood was similar, it implies that the two strains had different pathogenic properties. Indeed, using a modified in vitro assay first developed by Scheld et al. ${ }^{[15]}$, we could 
demonstrate that the $S$. faecalis strain adhered significantly more to platelet-fibrin matrices in vitro than the viridans streptococcal strain. Others have shown similar results in vitro using punch biopsy sections of human or canine aortic valve endothelium ${ }^{[16]}$.

More importantly, our recent studies on the production of endocarditis in rats after the extraction of periodontally diseased teeth have failed to demonstrate a correlation between the total number of a given streptococcal species that circulated immediately after teeth extractions and the likelihood that these streptococci will subsequently produce endocarditis ${ }^{[17]}$. Indeed, some streptococcal strains that circulated at barely detectable levels consistently produced endocarditis, while other viridans streptococci that were found in much higher numbers in the blood after extractions only rarely infected the valves. In these experiments, we could also show that the stickiness in vitro of a given bacterial species for platelet-fibrin matrices predicted best the likelihood that this strain would produce IE. Thus the determination of the magnitude of bacteraemia after certain procedures alone is unlikely to provide reliable information on the risk of subsequent development of IE.

\section{Conclusions}

Experimental models of IE were developed as a consequence of careful clinical observations. The disease produced in experimental models is much like the disease observed in humans. Moreover, the role of an intravascular foreign body can be accurately investigated. Experimental models of bacterial endocarditis offer several advantages for studying the prevention of the disease; proper experimental controls can be used and large numbers of animals can be studied in order to achieve statistically significant results.

More importantly, the model permits comparison of the efficacy of various antibiotic regimes against different organisms in vivo and will help unravel the mode of action of prophylactic antibiotics, thus giving a rationale for endocarditis prophylaxis recommendations in humans which may provide a wide margin of safety.

\section{References}

[1] Durack DT. Prophylaxis of infective endocarditis. In: Mandell GL, Douglas RG, Bennett JE, eds. Principles and practice of infectious diseases. New York: John Wiley and Sons, 1985: 539-44.
[2] Smits H, Freedman LR. Prolonged venous catheterization as a cause of sepsis. N Engl J Med 1967; 276: 1229-33.

[3] Durack DT, Beeson PB. Experimental bacterial endocarditis. I. Colonization of a sterile vegetation. $\mathrm{Br}$ J Exp Pathol 1972; 53: 44-9.

[4] Garrison PK, Freedman LR. Experimental endocarditis. I. Staphylococcal endocarditis in rabbits resulting from placement of a polyethylene catheter in the right side of the heart. Yale J Biol Med 1970; 42: 394-410.

[5] Heraief E, Glauser MP, Freedman LR. Vancomycin prophylaxis of streptococcal endocarditis in rats. In: Nelson JD, Grassi C, eds. Current chemotherapy and infectious disease. Washington DC: American Society of Microbiology, 1980: 911-3.

[6] McGowan DA. Scanning electron microscopy of heart valve vegetations. Lancet 1977; 2: 822 .

[7] Petersdorf RG. Antimicrobial prophylaxis of bacterial endocarditis. Prudent caution or bacterial overkill? Am J Med 1978; 65: 220-3.

[8] Francioli P, Freedman LR. Streptococcal infection of endocardial and other intravascular vegetations in rabbits: natural history and effects of dexamethasone. Infect Immun 1979; 24: 483-91.

[9] Perlman BB, Freedman LR. Experimental endocarditis. III. Natural history of catheter induced endocarditis after catheter removal. Yale J Biol Med 1971; 44: 214-23.

[10] Everett ED, Hirschmann JV. Transient bacteremia and endocarditis prophylaxis. A review. Medicine (Baltimore) $1977 ; 56: 61-77$.

[11] Bernard JP, Francioli P, Glauser MP. Vancomycin prophylaxis of experimental Streptococcus sanguis endocarditis. Inhibition of bacterial adherence rather than bacterial killing. J Clin Invest 1981; 68: 1113-6.

[12] Glauser MP, Francioli P. Successful prophylaxis of experimental streptococcal endocarditis with bacteriostatic antibiotics. J Infect Dis 1982; 146: 806-10.

[13] Glauser MP, Bernard JP, Moreillon P, Francioli P Successful single-dose amoxicillin prophylaxis against experimental streptococcal endocarditis: evidence for two mechanisms of protection. J Infect Dis 1983; 147: 568-75.

[14] Francioli P, Moreillon P, Glauser MP. Comparison of single doses of amoxicillin or of amoxicillin-gentamicin for the prevention of endocarditis caused by Streptococcus faecalis and by viridans streptococci. J Infect Dis 1985; 152: 83-9.

[15] Scheld WM, Valone JA, Sande MA. Bacterial adherence in the pathogenesis of endocarditis. Interaction of bacterial dextran, platelets and fibrin. J Clin Invest 1978; 61: 1394-404.

[16] Gould K, Ramirez-Ronda CH, Holmes RK, Sandford JP. Adherence of bacteria to heart valves in vitro. J Clin Invest 1975; 56: 1364-70.

[17] Moreillon P, Overholser CD, Bille J, Entenza J, Malinverni R, Glauser MP. Natural history of experimental bacterial endocarditis following dental extractions in rats with periodontitis (Abstr 465). In: Program and Abstracts of the 25th Interscience Conference on Antimicrobial Agents and Chemotherapy. Washington, DC: American Society for Microbiology, 1985. 